\title{
Hospitalist-Operated Compression Ultrasonography: a Point-of-Care Ultrasound Study (HOCUS-POCUS)
}

\author{
Ernest A. Fischer, $M D^{7}$, Benjamin Kinnear, $M D^{2,3}$, Dana Sall, $M D^{2}$, \\ Matthew Kelleher, MD ${ }^{2,3}$, Otto Sanchez, MD PhD', Benji Mathews, MD ${ }^{4,5}$, \\ Daniel Schnobrich, $M D^{4,6}$, and Andrew P. J. Olson, $M D^{4,6}$
}

'Department of Medicine, MedStar Georgetown University Hospital, Washington, DC, USA; ${ }^{2}$ Department of Medicine, University of Cincinnati, Cincinnati, OH, USA; ${ }^{3}$ Department of Pediatrics, University of Cincinnati, Cincinnati, OH, USA; ${ }^{4}$ Department of Internal Medicine, University of Minnesota Medical School, Minneapolis, MN, USA; ${ }^{5}$ HealthPartners, Minneapolis/St. Paul, MN, USA; ${ }^{6}$ Department of Pediatrics, University of Minnesota Medical School, Minneapolis, MN, USA.

BACKGROUND: Venous thromboembolism includes deep vein thrombosis (DVT) and pulmonary embolism. Compression ultrasonography is the most common way to evaluate DVT and is typically performed by sonographers and interpreted by radiologists. Yet there is evidence that ultrasound examinations can be safely and accurately performed by clinicians at the bedside.

OBJECTIVE: To measure the operating characteristics of hospital medicine providers performing point-of-care ultrasound (POCUS) for evaluation of DVT.

DESIGN: This is a prospective cohort study enrolling a convenience sample of patients. Hospital medicine providers performed POCUS for DVT and the results were compared with the corresponding formal vascular study (FVS) interpreted by radiologists.

PARTICIPANTS: Hospitalized non-ICU patients at four tertiary care hospitals for whom a DVT ultrasound was ordered.

MAIN MEASURES: The primary outcomes were the sensitivity, specificity, and predictive values of the POCUS compression ultrasound compared with a FVS. The secondary outcome was the elapsed time between order and the POCUS study compared with the time the FVS was ordered to when the formal radiology report was finalized. KEY RESULTS: One hundred twenty-five limbs from 73 patients were scanned. The prevalence of DVT was $6.4 \%$ $(8 / 125)$. The sensitivity of POCUS for DVT was $100 \%(95 \%$ CI 74-100\%) and specificity was 95.8\% (95\% CI 91-98\%) with a positive predictive value of $61.5 \%$ (95\% CI 35-84\%) and a negative predictive value of $100 \%$ (95\% CI 98 $100 \%)$. The median time from order to POCUS completion was $5.8 \mathrm{~h}$ versus $11.5 \mathrm{~h}$ median time from order until the radiology report was finalized $(p=0.001)$.

CONCLUSION: Hospital medicine providers can perform compression-only POCUS for DVT on inpatients with accuracy similar to other specialties and settings, with results available sooner than radiology. The observed prevalence of DVT was lower than expected. POCUS may be reliable in excluding DVT but further study is required to determine how to incorporate a positive POCUS DVT result into clinical practice.

Received April 30, 2018

Revised October 26, 2018

Accepted April 20, 2019

Published online August 6, 2019
KEY WORDS: ultrasonography; thromboembolism; hospitalists.

J Gen Intern Med 34(10):2062-7

DOI: $10.1007 / \mathrm{s} 11606-019-05120-5$

(C) Society of General Internal Medicine 2019

\section{INTRODUCTION}

Venous thromboembolism (VTE), including deep venous thrombosis (DVT) and pulmonary embolism (PE), is a major public health problem. Inpatient rates of incident and recurrent VTE range from 142 to $300+$ per 100,000 person years versus 8 per 100,000 in community-dwelling patients. ${ }^{1,2}$ VTE increases morbidity, mortality, and cost in hospitalized patients, ${ }^{3}$ and incidence of VTE has not improved with prophylaxis. ${ }^{4}$

Prompt recognition of DVT is paramount to reduce PE risk, ${ }^{5}$ yet diagnostic strategies remain suboptimal. While risk factors are known and guidelines delineate indications for ordering diagnostics, ${ }^{6}$ no prospectively validated risk stratification for inpatient DVT exists. For example, Wells score performs poorly on inpatients. ${ }^{7}$

DVT is typically diagnosed using ultrasonography: either compression (CUS) alone, duplex (CUS plus color Doppler), or triplex (duplex with continuous wave Doppler). One metaanalysis of radiology performed CUS reported a sensitivity of 94\% for proximal DVTs and $57 \%$ for distal DVTs with an overall specificity of $98 \% .^{8}$ CUS has reduced accuracy with recurrent DVT but is considered appropriate for a suspected first episode. 8,9

CUS can be performed as proximal only (femoral and popliteal veins) or whole-leg (proximal and distal veins). Serial two-point CUS (1-week apart) of the proximal venous system is equivalent to whole-leg examination for suspected first episode of DVT, ${ }^{10,11}$ and proximal vein CUS is recommended as an initial test for suspected first episode of lower extremity DVT. ${ }^{12}$ Despite this, formal ultrasounds are not universally available or technologists may only be available during weekday daytime hours, delaying formal read, diagnosis, ${ }^{13,14}$ and potentially lifesaving anticoagulation. 
Point-of-care ultrasound (POCUS) has emerged for rapid clinician diagnosis of DVT. Emergency Medicine (EM) physicians evaluated POCUS to diagnose DVT and reported a sensitivity and specificity of $96.1 \%$ and $96.8 \%$, respectively. ${ }^{15}$ General practitioners had a sensitivity of $90 \%$ and specificity of $97.1 \%$ when assessing outpatients. ${ }^{16}$ In the ICU, critical care physicians had a sensitivity ranging $63-85 \%$ and specificity $96-97 \%$ for DVT diagnosis. ${ }^{13,14}$ The variable DVT prevalence in these populations impacted positive and negative predictive values. In the Caronia et al. ICU study, the superficial femoral vein was not scanned, nor was the popliteal vein from its most proximal to its most distal extent. The authors attributed their lower sensitivity $(63 \%)$ to these exclusions. ${ }^{13}$

The Hospitalist-Operated Compression Ultrasound Study - A Point-of-Care Ultrasound Study (HOCUSPOCUS) is a prospective, multi-centered trial to determine the accuracy and feasibility of hospitalist-performed POCUS in DVT evaluation of hospitalized, non-ICU patients. To our knowledge, POCUS for DVT diagnosis has not been evaluated on this population. We utilized a threeregion compression protocol to attempt to identify all proximal DVTs. The primary outcomes of the study are the operating characteristics (sensitivity and specificity) of hospitalists performing DVT POCUS compared with radiology formal vascular studies (FVS) as the reference standard.

\section{METHODS}

\section{Study Design and Population}

This multicenter study was conducted at four institutions: The University of Minnesota, MedStar Georgetown University, HealthPartners/Regions, and The University of Cincinnati Medical Center. Each site's Institution Review Board approved the study. Patients were enrolled between May 2016 and June 2017.

Adults ( $\geq 18$ years) were eligible for inclusion if they were admitted to a non-ICU general medicine ward and had a high enough pre-test probability that the primary team ordered a lower extremity DVT ultrasound of either or both legs. Patients were excluded if they (1) had critical illness precluding examination, (2) had DVT diagnosed within 90 days, (3) had pain limiting examination, (4) were unable to consent, or (5) were non-English speaking.

The study patients represent a convenience sample based on hospitalist availability. Hospitalist scanners were not necessarily contacted at the time of a FVS order and were never aware of FVS results prior to performing POCUS. Sonographers independently chose scanning days and each scanning day the ordering primary team would contact the hospitalist scanner, or the sonographer would use the medical record to find new studies. Once identified, the protocol and FVS were completed regardless of the result (i.e., a negative POCUS could not be used to cancel the FVS). Neither potentially eligible nor excluded patients were tracked. Study flow is summarized in Figure 1.

\section{Outcomes and Statistical Analysis}

The pre-specified primary outcomes were the sensitivity and specificity of the POCUS CUS compared with a "gold standard" FVS, either the radiology performed duplex or triplex. The FVS is a practical standard as direct or CT/MRI venography is not routinely performed and duplex/triplex has excellent accuracy for proximal DVT. ${ }^{8}$ We recorded the time the POCUS was performed for comparison with the time the FVS was ordered and time the formal radiology report became available.

POCUS and FVS results were treated as dichotomous outcomes. POCUS study results were recorded as compressible or not, while for FVS, results were recorded as DVT present or absent. For safety, any noncompressibility was recorded as "positive" regardless of the hospitalist's suspicion for DVT. If an area was not visualized, this was noted but the study was still included in the analysis. For statistical analysis, legs were counted independently (a bilateral study was treated as two separate scans).

Based on previous literature, we expected the prevalence of DVT in the eligible population to be $22 \% .^{13-16}$ We anticipated POCUS to have a minimum sensitivity of $95 \%$, requiring 317 extremities to provide a confidence interval of $5 \% .{ }^{17}$ To reach the goal, each of the four centers would scan approximately 80 extremities. We did not meet this goal due to slow accrual and the study was stopped after 125 extremities.

All data were entered into the RedCap database. All POCUS data were recorded at the bedside and formal vascular study results and clinical information were documented. The elapsed times from FVS order to POCUS completion and to FVS report completion were tabulated. These were compared via the Wilcoxon signed-rank test. Performance of diagnostic testing was assessed by sensitivity, specificity, negative and positive predictive values (NPV and PPV, respectively), and negative and positive likelihood ratios, ( $\mathrm{LR}+$ and $\mathrm{LR}-$, respectively). The $95 \%$ confidence intervals were calculated using the Bayesian Jeffreys method. ${ }^{18,19}$ Statistical analyses were performed using SAS 9.4 (Copyright 2002-2012, SAS Institute Inc., Cary, NC, USA) and a Clinical Decision Making Spreadsheet Calculator designed by Robert M. Hamm (oumedicine.com/familymedicine/academic-information/ research/research-faculty/rob-hamm/download-computerprograms-for-cdm-calculations/cdm-calculators, accessed September, 2018). 


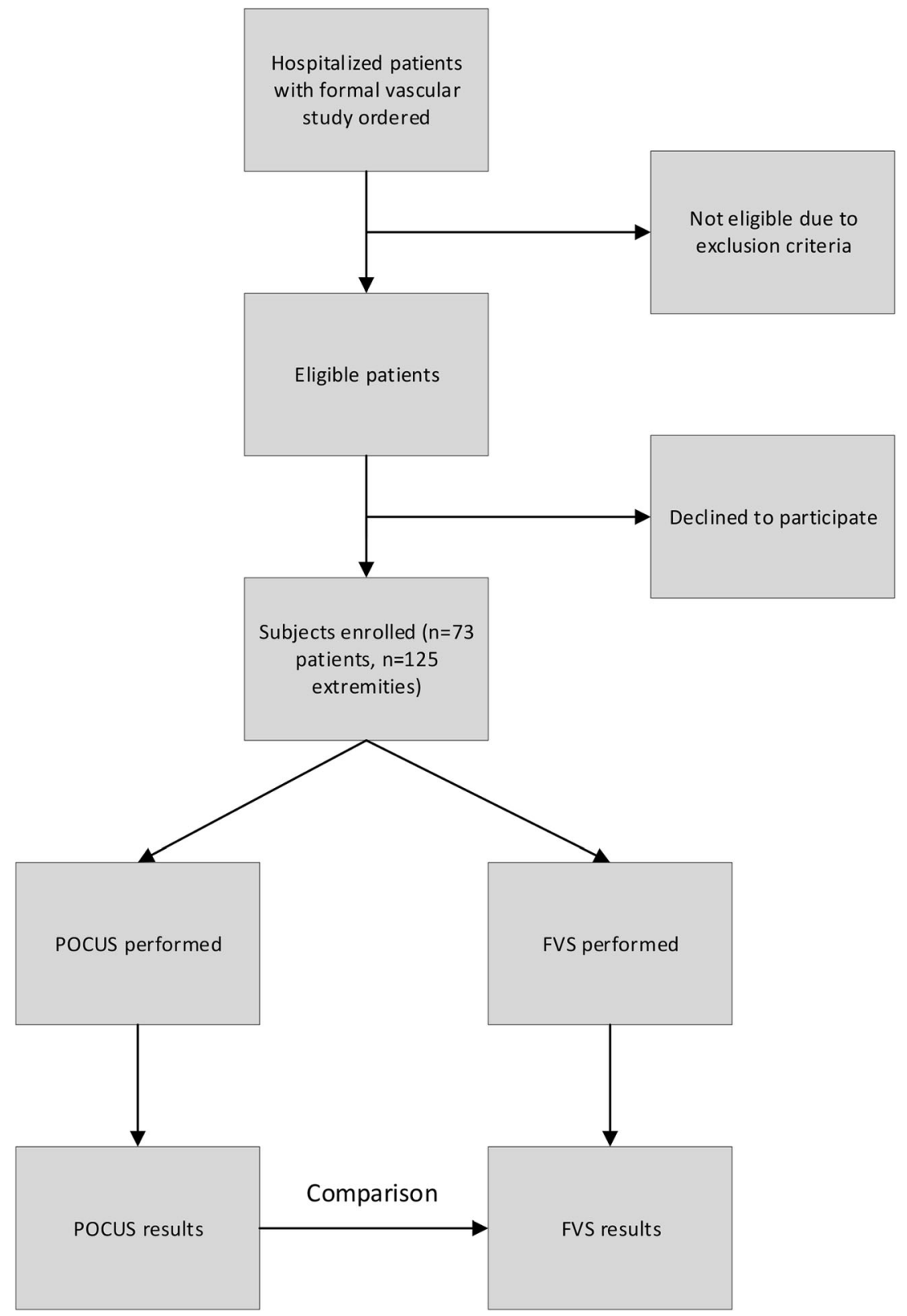

Fig. 1 HOCUS-POCUS study design and flow.

\section{Ultrasound Training and Proficiency}

All participating hospitalist scanners completed didactic and hands-on training ${ }^{20}$ totaling $2 \mathrm{~h}$. Each hospitalist had to successfully complete ten normal proctored DVT studies on standardized patients in order to begin enrolling patients. The didactic contained "abnormal" examples.

\section{POCUS Protocol}

POCUS was standardized as follows: The hospitalist scanner begins at the common femoral vein and compresses, moves distally both noting and compressing the greater saphenous, the common femoral arterial bifurcation, and venous bifurcation (zone 1) before reaching the proximal superficial femoral vein (SFV, zone 2). Our protocol includes the entire SFV that is visible with a standard linear probe. The sonographer proceeds distally along the SFV compressing at $1-2-\mathrm{cm}$ intervals until it is no longer visible, a variable distance dependent on patient anatomy. Finally, the popliteal vein is compressed (zone 3). Color or spectral Doppler were not used.

\section{Ultrasound Machines}

Each institution used the equipment available to them. MedStar Georgetown used a Sonosite M-turbo provided by the company for the study as well as a Sonosite Nanomaxx. The University of Cincinnati used a Sonosite S-Fast; HealthPartners used Sonosite Edge I, II, and Export; and the University of Minnesota used a MindRay T-7. 
Table 1 Baseline Characteristics of Patients Enrolled

\begin{tabular}{ll}
\hline \hline Characteristic & Value \\
\hline Age, years (median, range) & $61(26-92)$ \\
BMI, kg/m² (mean, standard deviation) & $31.4(8.4)$ \\
Male sex, \% & 49 \\
Primary discharge diagnoses $(n)$ & 15 \\
Other* & 14 \\
Infection & 9 \\
Cancer & 8 \\
Respiratory & 8 \\
VTE & 7 \\
Cardiac/heart failure & 4 \\
AKI/ESRD & 4 \\
Trauma/wound & 2 \\
CVA/ICH & 2 \\
Hepatic/cirrhosis &
\end{tabular}

*Other includes the following: abnormal vaginal bleeding, bilateral LE swelling, chest pain, complication of dialysis catheter, DKA, frequent falls, hypertensive emergency, hyponatremia, leukocytosis, periumbilical abdominal pain, pleuritic chest pain, right pneumothorax, SBO, UC flare, vocal cord dysfunction

\section{RESULTS}

Eight providers performed the POCUS DVT assessments across the four institutions.

A total of 73 patients representing 125 extremities were enrolled. Subject characteristics are shown in Table 1. The hospitalists had performed between 2 and 40 studies prior to the required training. The prevalence of DVT was $6.4 \%$ of limbs scanned (8/125) and $8.2 \%$ $(6 / 73)$ of patients. Bilateral studies made up the majority of those ordered $(71.2 \%, 52 / 73)$. In patients with bilateral studies ordered, the prevalence was $9.6 \%(5 / 52)$. In 21 patients with unilateral studies, only one DVT was found. The POCUS results compared with FVS are summarized in Table 2. When compared with the radiology FVS, the sensitivity of hospitalist-performed POCUS for DVT was $100 \%$ (95\% CI $74-100 \%)$ and the specificity was $95.8 \%$ (95\% CI $90-98 \%$ ). Table 3 shows all operating characteristics of the hospitalistperformed POCUS. Details for the five false positive studies are shown in Table 4.

There was no statistical difference in the median time from FVS order to POCUS completion versus time to technician performing the FVS (5.8 vs $4.7 \mathrm{~h}, p=0.36$ ); however, the POCUS exam was completed before the FVS report $68 \%$ of the time. The median time until the FVS report became available was $11.5 \mathrm{~h}$, significantly longer than POCUS completion $(p=0.001)$.

Table 2 Two by Two Table of Results Showing Bedside Sonographer POCUS Findings Compared with the Formal Vascular Study

\begin{tabular}{llll}
\hline \hline & \multicolumn{2}{l}{ Formal vascular study } \\
\cline { 3 - 4 } & & DVT present & DVT absent \\
\hline POCUS result & Not compressible & 8 & 5 \\
& Compressible & 0 & 112 \\
\hline
\end{tabular}

Table 3 HOCUS-POCUS Study Outcomes Including Operating Characteristics of Hospitalist-Performed CUS for Diagnosis of DVT

\begin{tabular}{lll}
\hline \hline Operating characteristic & Value & 95\% CI \\
\hline Prevalence (125 extremities) & $6.4 \%$ & \\
Prevalence (73 patients) & $8.2 \%$ & \\
Sensitivity & $100 \%$ & $74-100 \%$ \\
Specificity & $95.8 \%$ & $91-98 \%$ \\
LR+ & 23.4 & $9.9-55.1$ \\
LR- & 0 & $0-0.32$ \\
PPV & $61.5 \%$ & $35-84 \%$ \\
NPV & $100 \%$ & $98-100 \%$ \\
\hline
\end{tabular}

NPV¥ A range for the post-test probability of DVT was assumed based on the 95\% CI for the NPV. Using this, the upper limit for 193 the LRconfidence interval could be estimated according to the Baves theorem $(L R=$ post-test odds/pre-test odds)

\section{DISCUSSION}

In HOCUS-POCUS, we have shown via a prospective, multicenter trial on hospitalized non-ICU patients that hospitalist performed POCUS for DVT has excellent specificity (95.7\%) and that results were available over $5 \mathrm{~h}$ before completed radiology reports. The sensitivity and specificity are similar to POCUS studies in the general outpatient clinic, ${ }^{16}$ emergency department, ${ }^{15}$ and ICU. ${ }^{13,14}$ Though our observed sensitivity was $100 \%$, the confidence interval does not exclude that the true sensitivity could have been as low as $74 \%$. Our negative predictive value of $100 \%$ (95\% CI 98-100\%) is also similar to others and suggests that trained hospitalists accurately exclude DVT in patients for whom there is reason to order an FVS. The $6.4 \%$ observed extremity prevalence of DVT resulted in a lower positive predictive value than previous POCUS studies $(61.5 \%$ vs others at $80-90 \%)$. Prior POCUS DVT study results are summarized in Table 5.

Our study did have a number of limitations. One was the sample size. We did not reach our enrollment goal due to slow enrollment and lower than expected FVS frequency. The study was stopped prematurely as it became clear that the false negative rate would be extremely low and that slow

Table 4 False Positive Results within HOCUS-POCUS

\begin{tabular}{ll}
\hline \hline Age, gender laterality & Description of findings \\
\hline 74-year-old male, & $\begin{array}{l}\text { 1. Unable to compress left mid SFV, } \\
\text { no clot identified. Unable to visualize left } \\
\text { distal SFV. } \\
\text { Patient sitting up in chair at time of exam; } \\
\text { back pain limited ability to optimally } \\
\text { position patient for duration of exam. }\end{array}$ \\
$\begin{array}{l}\text { 2. Unable to compress branch point tributary } \\
\text { near R CFV bifurcation. }\end{array}$ \\
$\begin{array}{l}\text { 36-year-old female, } \\
\text { unilateral (right) }\end{array}$ \\
$\begin{array}{l}\text { b8-year-old female, } \\
\text { unilateral (right) }\end{array}$ & $\begin{array}{l}\text { low suspicion of true DVT as the vein } \\
\text { immediately proximal was almost fully } \\
\text { compressible and appeared issue seemed } \\
\text { related to artifact, but this was called a } \\
\text { positive exam due to the predetermined } \\
\text { criteria. } \\
\text { 4. Left: dialysis fistula present, unable to } \\
\text { compress } \\
\text { 5. Right: examiner indicated visible clot }\end{array}$ \\
\end{tabular}


Table 5 Summary of HOCUS POCUS and other POCUS DVT Study Outcomes Including Operating Characteristics

\begin{tabular}{lllllll}
\hline \hline Study & Location & $\boldsymbol{n}$ & Prevalence & Sensitivity & Specificity & PPV \\
\hline HOCUS-POCUS & Wards & 125 & 6.4 & 100 & 95.7 & 61.5 \\
PRACTICUS $^{16}$ & Outpatient & 1107 & 18.1 & 90 & 97.1 & 87.4 \\
PD Kory, et al. $^{14}$ & ICU & 128 & 20 & 86 & 96 & 97.8 \\
J Caronia, et al. $^{13 *}$ & ICU & 75 & 16 & $63+$ & 97 & 94.3 \\
F. Pomero, et al. $^{15 \dagger}$ & ED & 2379 & 23.1 & 96.1 & 96.8 & 90.5 \\
\hline
\end{tabular}

*Used a 2-point compression protocol and missed SFV and possibly popliteal DVTS

†Meta-analysis of 16 cohorts

enrollment would preclude timely completion. We do not believe the operating characteristics would significantly differ had we reached our goal, though the confidence intervals would likely be narrower.

Additionally, patients were only enrolled during prespecified scanning days, leading to a convenience rather than consecutive sample. Yet median POCUS results were available over $5 \mathrm{~h}$ before median FVS reports. To limit selection bias, scanners chose scanning days in advance, and all eligible patients were approached to participate. Still, most scans were performed during the weekday, daytime hours, and it is possible that this patient subset differed from that during offhours. Even though we cannot say included patients were randomly selected, there was no pattern to our scanning days and no reason to believe this led to a population bias, though it may limit generalizability.

We assumed observations were independent. Limbs are not biologically independent but it is unclear if they are statistically independent. Of the five positive bilateral studies, three $(60 \%)$ were positive on only one limb. There are other potential factors that could have led to non-independent observations such as the scanner, institution, or sonographer experience. Our study was too small to explore these.

As we did not want to influence a patient's decision to proceed with a FVS, we excluded patients with significant pain, though these patients could have a higher prevalence of DVT. Furthermore, it is possible that patients with pain or higher degree of illness were more likely to decline participation. In contrast, we did not exclude those with prior DVT as part of the pragmatic study design, even though CUS has reduced accuracy in recurrence. Duplex or triplex is still the FVS ordered for recurrent DVT in the inpatient setting, and we were comparing hospitalist-performed POCUS to real-world practice.

The positive predictive value $(61.5 \%)$ was lower than that in previous studies, owing to the lower DVT prevalence in our study. Our scanning protocol favored sensitivity over specificity, requiring categorical responses (compressible, noncompressible, or visualized clot) independent of the scanner's clinical suspicion. Though not representative of typical POCUS practice, this decision allowed evaluation of hospitalist POCUS technique and prioritized not missing critical DVT diagnoses. Not surprisingly, there were false positives in the study; however, many probably would not have been deemed positive in practice (see Table 4). We believe additional review of clinical information would have decreased the pre-test probability of DVT or even negated some FVS orders (i.e., bilateral FVS for fever or bilateral swelling in heart failure).

The low overall DVT prevalence in our sample was unexpected. The predicted DVT prevalence was consistent with outpatient, ED, and ICU studies (see Table 5) and published FVS rates. However, FVS protocols often include distal deep veins and distal DVTs represent nearly half of all positive results. ${ }^{12}$ Additionally, many FVSs are obtained in the high pretest scenario of known VTE (i.e., known PE). Known VTE and concern for distal DVT are not always scenarios to implement POCUS, which we advocate should be primary for rapid assessment that impacts clinical decision-making. We suspect the PPV would have been higher in a pragmatic study design in which the hospitalist could choose subjects based on pretest probability. The logistics of such a study would invariably be more difficult.

\section{CONCLUSIONS}

HOCUS-POCUS adds to the literature on POCUS for DVT in several ways. It is the first study in non-ICU hospitalized patients and shows that hospitalists can be trained to competently perform POCUS with good sensitivity and excellent specificity. Second, the use of a three-region compression protocol as opposed to more common 2- or 3-point compression protocols resulted in no false negatives and a high NPV. Two-point compression protocols, though possibly sufficient in ambulatory patients, have missed clinically significant DVTs in the SFV in inpatients ${ }^{13}$.

In addition to establishing operating characteristics, this study showed the feasibility of training hospitalists in POCUS for DVT. Our hospitalists received only $2 \mathrm{~h}$ of training and completed ten practice scans on standardized patients. Many of the hospitalist scanners were new to POCUS or had no previous formal training. The training is both relatively fast and reproducible with minimal resources.

POCUS is an increasingly important skill for the modern hospitalist and we foresee the results from this study informing hospital medicine practice in myriad ways: (1) we present a learnable, effective protocol that improves decision-making for hospitalists working in settings where FVS cannot be obtained or interpreted in a reasonable timeframe, (2) this 
protocol is safe and accurate, (3) our findings suggest that a negative POCUS scan could be sufficient to rule out DVT in a hospitalized patient, and (4) there is potential to develop a clinical workflow and encourage hospitalists to consider other pathologies after DVT is excluded via POCUS, before or possibly without a FVS. Further study is needed to ensure generalizability of our findings, understand how to integrate this into a hospitalist's workflow, and consider how this approach might be shared with trainees.

Acknowledgments: The authors wish to acknowledge all of their colleagues who participated in this study by performing POCUS scans.

Corresponding Author: Ernest A. Fischer, MD; Department of Medicine MedStar Georgetown University Hospital, Washington, DC, USA (e-mail: Ernest.A.Fischer@gunet.georgetown.edu).

\section{Compliance with Ethical Standards:}

Conflict of Interest: The authors declare that they have no conflict of interest.

\section{REFERENCES}

1. Heit JA, Spencer FA, White RH. The Epidemiology of Venous Thromboembolism. J Thromb Thrombolysis. 2016;41(1):3-14. https://doi.org/ 10.1007/s11239-015-1311-6.

2. Heit JA. Epidemiology of Venous Thromboembolism. Nat Rev Cardiol. 2015; 12(8):464-474. https://doi.org/10.1038/nrcardio.2015.83.

3. DHHS. The surgeon general's call to action to prevent deep vein thrombosis and pulmonary embolism. 2008. http://www.ncbi.nlm.nih. gov/pubmed/20669525. Accessed 2/9/2019.

4. Heit JA, Crusan DJ, Ashrani AA, Petterson TM, Bailey KR. Effect of a Near-Universal Hospitalization-Based Prophylaxis Regimen on Annual Number of Venous Thromboembolism Events in the US. Blood. 2017;130(2): 109-114. https://doi.org/10.1182/blood-2016-12-758995.

5. Kearon C, Akl EA, Comerota AJ, et al. Antithrombotic Therapy for VTE Disease: Antithrombotic Therapy and Prevention of Thrombosis, 9th ed: American College of Chest Physicians Evidence-Based Clinical Practice Guidelines. Chest. 2012;141(2 Suppl):e419S-e496S. https://doi.org/10 1378/chest.11-2301.

6. Gornik HL, Gerhard-Herman MD, Misra S, et al. ACCF/ACR/AIUM/ ASE/IAC/SCAI/SCVS/SIR/SVM/SVS/SVU 2013 Appropriate Use Criteria for Peripheral Vascular Ultrasound and Physiological Testing Part II: a Report of the American College of Cardiology Foundation Appropriate Use Criteria Task Force. J Am Coll Cardiol. 2013;62(7):649665. https://doi.org/10.1016/j.jacc.2013.05.001

7. Silveira PC, Ip IK, Goldhaber SZ, Piazza G, Benson CB, Khorasani R. Performance of Wells Score for Deep Vein Thrombosis in the Inpatient
Setting. JAMA Intern Med. 2015;175(7):1112-1117. https://doi.org/10. 1001/jamainternmed.2015.1687.

8. Goodacre S, Sampson F, Thomas S, van Beek E, Sutton A. Systematic Review and Meta-analysis of the Diagnostic Accuracy of Ultrasonography for Deep Vein Thrombosis. BMC Med Imaging. 2005;5:6. https://doi.org/ 10.1186/1471-2342-5-6.

9. Huisman M V, Klok FA. Current Challenges in Diagnostic Imaging of Venous Thromboembolism. Blood. 2015;126(21):2376-2382. https:// doi.org/10.1182/blood-2015-05-640979

10. Bernardi E, Camporese G, Büller HR, et al. Serial 2-Point Ultrasonography Plus D-dimer vs Whole-Leg Color-Coded Doppler Ultrasonography for Diagnosing Suspected Symptomatic Deep Vein Thrombosis: a Randomized Controlled Trial. JAMA. 2008;300(14):1653-1659. https://doi. org/10.1001/jama.300.14.1653.

11. Gibson NS, Schellong SM, Kheir DY El, et al. Safety and Sensitivity of Two Ultrasound Strategies in Patients with Clinically Suspected Deep Venous Thrombosis: a Prospective Management Study. J Thromb Haemost 2009;7(12):2035-2041. https://doi.org/10.1111/j.1538-7836. 2009.03635.x.

12. Bates SM, Jaeschke R, Stevens SM, et al. Diagnosis of DVT: Antithrombotic Therapy and Prevention of Thrombosis, 9th ed: American College of Chest Physicians Evidence-Based Clinical Practice Guidelines. Chest. 2012;141(2 SUPPL). https://doi.org/10.1378/chest.11-2299.

13. Caronia J, Sarzynski A, Tofighi B, et al. Resident Performed Two-Point Compression Ultrasound is Inadequate for Diagnosis of Deep Vein Thrombosis in the Critically III. J Thromb Thrombolysis. 2014;37(3):298-302. https://doi.org/10.1007/s11239-013-0945-5.

14. Kory PD, Pellecchia CM, Shiloh AL, Mayo PH, DiBello C, Koenig S. Accuracy of Ultrasonography Performed by Critical Care Physicians for the Diagnosis of DVT. Chest. 2011;139(3):538-542. https://doi.org/10. 1378/chest. 10-1479.

15. Pomero F, Dentali F, Borretta V, et al. Accuracy of Emergency Physician-Performed Ultrasonography in the Diagnosis of Deep-Vein Thrombosis: a Systematic Review and Meta-Analysis. Thromb Haemost 2013;109(1):137-145. https://doi.org/10.1160/TH12-07-0473.

16. Mumoli N, Vitale J, Giorgi-Pierfranceschi $\mathbf{M}$, et al. General Practitioner-Performed Compression Ultrasonography for Diagnosis of Deep Vein Thrombosis of the Leg: a Multicenter, Prospective Cohort Study. Ann Fam Med. 2017;15(6):535-539. https://doi.org/10.1370/ afm.2109.

17. Jones SR, Carley S, Harrison M. An Introduction to Power and Sample Size Estimation. Emerg Med J. 2003;20(5):453-458. https://doi.org/10. $1136 / \mathrm{emj} .20 .5 .453$

18. Jeffreys $\mathbf{H}$. An invariant form for the prior probability in estimation problems. Proc R Soc Lond A Math Phys Sci. 1946;186(1007):453-461. http://www.ncbi.nlm.nih.gov/pubmed/20998741.

19. Brown LD, Cai TT, DasGupta A. Interval estimation for a binomial proportion. Stat Sci 2001;16(2):101-133.

20. Schnobrich DJ, Olson AP, Broccard A, Duran-Nelson A. Feasibility and Acceptability of a Structured Curriculum in Teaching Procedural and Basic Diagnostic Ultrasound Skills to Internal Medicine Residents. J Grad Med Educ. 2013;5(3):493-497. https://doi.org/10.4300/JGME-D$12-00214.1$

Publisher's Note: Springer Nature remains neutral with regard to jurisdictional claims in published maps and institutional affiliations. 